\title{
Uma Abordagem de Gestão e Desenvolvimento de Automatização de Processos de Negócios com apoio de BPMS
}

\author{
Luana P. Ramos ${ }^{1}$, Adriano Bessa ${ }^{1}$ \\ ${ }^{1}$ Programa de Pós-Graduação em Informática Aplicada (PPGIA) \\ Universidade de Fortaleza (UNIFOR) - Fortaleza, CE - Brasil 60.811-905 \\ luana.pires@gmail.com, adrianoba@unifor.br
}

\begin{abstract}
In response to current challenges, to align the BPM (Business Process Management) discipline, Software Engineering concepts, SCRUM, Agile Software Development, as well as SOA (Service Oriented Architecture) and understanding between Process, Business and IT areas, this work presents a software development approach to automate business processes with BPMS (Business Process Management System) support. The approach is both a guide for IT professionals develop business process-oriented software systems and for business professionals take ownership of systems thinking and the steps required to automation. The development and validation of the approach were performed through action research projects.
\end{abstract}

Resumo. Em resposta aos desafios em aliar conceitos da disciplina de BPM (Business Process Management) aos conceitos de Engenharia de Software, SCRUM, desenvolvimento ágil de software, assim como conceitos de SOA (Service Oriented Architecture) e entendimento entre as áreas de Processos, Negócios e TI, este trabalho apresenta uma abordagem de desenvolvimento de software, para automatizar processos com apoio de BPMS (Business Process Management System). A abordagem é um guia para os profissionais de TI e de negócio para que ambos se apropriem dos passos necessários ao desenvolvimento de automatização e desenvolvam uma solução de forma orientada a processos. A abordagem foi validada através de projetos de pesquisa-ação.

\section{Introdução}

A forma de pensar, transformar e implementar processos, de uma forma geral, desde sempre evolui e vem abrangendo diversas metodologias no âmbito de melhoria (Six Sigma, Lean, TQM, Custeio baseado em atividade e gerenciamento baseado em atividade), de reengenharia e de redesenho de processos. Tais metodologias ao longo dos anos foram aplicadas em processos de negócio nos setores primários, secundários e terciários [Harmon 2010].

Em 2003, vários produtos surgiram com o objetivo de fornecer suporte ao ciclo de processos de negócio e à implementação da estratégia através da operacionalização de processos, cobrindo desde o desenho até a implementação, incluindo execução e controle de processos. O conjunto de tecnologias/ferramentas desenvolvidas é chamado de BPMS (Business Process Management Suite). Dessa forma, as aplicações de software desenvolvidas, com apoio do BPMS para automatizar processos de negócio, tornou-se um compo- 
nente estratégico para diversas áreas de negócio, apoiando a execução e o gerenciamento das várias instâncias em execução do processo [ABPMP 2013].

\subsection{Problema}

O nível de formação de grande parte dos profissionais de TI e de negócio que precisam trabalhar com BPMS e com a disciplina de BPM, em geral, é um fator desafiador. O tema BPM ainda tem sido pouco abordado em Universidades, em especial, nos cursos relacionados a Sistemas de Informação no Brasil, sendo mais abordado nos cursos de Administração e Engenharia de Produção. Geralmente, os profissionais de TI começam o desenvolvimento da automatização pela análise de funcionalidades sistêmicas e dentro de uma perspectiva tecnológica, em vez de realizar um desenvolvimento orientado por processos de negócio, visando atingir os objetivos estratégicos da organização. Dessa forma, os profissionais de TI precisam se apropriar mais da disciplina de gerenciamento de processos para melhor contribuir com o planejamento e as mudanças que serão realizadas nos processos. Assim como os profissionais de negócio precisam desenvolver o pensamento sistêmico e ter a compreensão clara do que, como e por que são feitas certas funcionalidades em nível de automatização de processos.

O processo de desenvolvimento de sistemas orientados a processos de negócio tem sido conduzido por diversas metodologias proprietárias ou "ad-hoc", ou seja, não padronizada. Quando uma organização não formaliza um processo de desenvolvimento, abre-se uma margem para erros, aumentando consideravelmente o risco de uma adoção mal sucedida de disciplinas, metodologias e práticas. Ter uma abordagem prescritiva do processo significa conhecer como os produtos e serviços são planejados, produzidos e entregues.

Os fatores citados acima, foram encontrados na instituição em estudo, ao longo de pesquisas-ação realizadas durante projetos, que tinham como objetivo gerar aplicações automatizadas de processo de negócio, com apoio de BPMS. As pesquisas-ação foram realizadas na sede de uma instituição pública financeira múltipla, de grande porte, organizada sob a forma de sociedade anônima aberta, na qual encontram-se os Ambientes de Negócio, Processos, Sistemas de Informação e Infraestrutura.

Outros fatores encontrados nas pesquisas-ação, foram a sensação de distância e dificuldades em coordenar os trabalhos de levantamento de requisitos de processo e, consequentemente, desenvolvimento da automatização, com equipes interfuncionais, que trabalhavam de forma distribuída. Esses problemas são também encontrados durante desenvolvimento de sistemas tradicionais [Audy and Prikladnicki 2007]. O trabalho distribuído se caracteriza por ter pessoas dispersas geograficamente, com diferenças socioculturais e, portanto, com diferentes níveis de dispersão entre elas. Quando a distância entre colaboradores distribuídos atinge trinta metros ou mais, a frequência de comunicação diminui para um nível idêntico ao de colaboradores que estão distribuídos a milhares de metros [Herbsleb et al. 2001].

Percebeu-se também, que os projetos não entregavam soluções de melhorias de processo, relacionadas a automatização, de acordo com o que foi solicitado pela estratégia da organização e em tempo hábil. Percebeu-se que isso acontecia porque a definição da aplicação para automatizar o processo era dirigida a funcionalidades sistêmicas, ou seja, o processo de negócio refletia sistema e não o contrário. Além disso, a aplicação não era 
projetada para permanecer preparada para mudanças rápidas. Quando o processo não é desenhado pensando nessa adaptabilidade ágil, os ciclos de entrega da área de Tecnologia da Informação, que implementam as funcionalidades de mudança, tem a dificuldade em seguir o ritmo de mudança dos negócios.

Contudo, o principal problema identificado foi a dificuldade em aliar conceitos da disciplina de BPM aos conceitos, ferramentas e metodologias já utilizadas no desenvolvimento tradicional de sistemas relacionados à Engenharia de Software, SCRUM [Schwaber and Beedle 2001], desenvolvimento ágil de sistemas, assim como conceitos de SOA [Erl 2008] e do próprio framework RUP (Rational Unified Process) [Kruchten 2004], mais especificamente no tocante às melhores práticas, planejamento/gestão das atividades e a colaboração eficiente entre áreas de Processo, Negócio e TI.

Dessa forma, diante da necessidade de constante evolução dos processos de desenvolvimento de soluções automatizadas, para prover melhores resultados na execução e gerenciamento dos processos de negócio, desenvolveu-se uma abordagem de processo para gestão e desenvolvimento de automatização para processos de negócio, cujo desenvolvimento da automatização é apoiado por BPMS.

\subsection{Trabalhos Relacionados}

Para buscar soluções para problemas identificados ao longo das pesquisas-ação, um levantamento dos trabalhos existentes e relacionados à gestão e desenvolvimento de sistemas (automatização) para apoiar processos de negócio foi realizado e representado na Tabela 1.

Identificou-se que, atualmente, existe uma variedade de metodologias com diferentes níveis de estrutura (fases, atividades, tarefas, funções, artefatos e ferramentas). Há algumas metodologias proprietárias bem estruturadas (ARIS, IBM, Oracle), porém não são acessíveis para quem não é proprietário do BPMS. Para se obter um comparativo entre os trabalhos que propuseram metodologias, abordagens e processos para desenvolvimento de automatização de processos, foi elaborada a Tabela 2, contendo as similaridades e as diferenças com a abordagem proposta nesse trabalho, representada na tabela pelo T0.

\section{Fundamentação}

Um dos principais conceitos do RUP utilizados na abordagem proposta é a noção de desenvolvimento iterativo. O RUP organiza projetos em termos de disciplinas e fases, cada uma consistindo em uma ou mais iterações. Ao especificar o processo de desenvolvimento da automatização, também definimos as fases: Iniciação, Elaboração, Construção e Transição como marcos, da mesma forma como se define marcos para processos de negócio em geral. De forma análoga ao RUP a abordagem descreve "quem" (papel) está fazendo "o que" (artefato), "como" (atividade) e "quando" (fluxo das atividades) [Kruchten 2004].

Não há, definido no RUP, uma obrigatoriedade de realizar todas as atividades e papéis prescritos nele. Na prática, projetos que envolvem desenvolvimento de software, adotam o RUP como guia e instanciam versões do RUP considerando o que se compatibiliza para atender às próprias necessidades. Dessa mesma forma, a abordagem obtida nesse trabalho, considera alguns elementos do RUP identificados como adequados para 


\begin{tabular}{|c|c|c|}
\hline Referência & Autores & Descrição \\
\hline $\mathbf{T 2}$ & $\begin{array}{l}\text { Vidales, Hermoso e Joyanes } \\
\text { [Vidales et al. 2006] }\end{array}$ & $\begin{array}{l}\text { Definem uma abordagem focada em contexto "On Demand Business" com base na Arquitetura Dirigida } \\
\text { a Modelo (MDA - Model Driven Architecture) e SOA. A metodologia compreende análise, modelagem } \\
\text { e simulação de processos de negócio. Além disso, abrange conversão de modelos CIM-PIM, definição } \\
\text { de requisitos a serem automatizados, identificação e análise de serviços. A abordagem é representada em } \\
\text { diagrama de atividades. }\end{array}$ \\
\hline T3 & $\begin{array}{l}\text { Papazoglou e Van Den } \\
\text { [Papazoglou and Van Den Heuvel 2007] }\end{array}$ & $\begin{array}{l}\text { Fornecem uma abordagem para o desenvolvimento de serviços para automatização de processos de negócio } \\
\text { em cinco fases. O foco principal está em definir um roteiro de desenvolvimento para web services dentro } \\
\text { do contexto de processos de negócio. }\end{array}$ \\
\hline T4 & An Liping et al. [An et al. 2008] & $\begin{array}{l}\text { Descreve uma metodologia sistemática para priorizar fatores que influenciam à adoção de web services } \\
\text { durante o desenvolvimento de automatização para processos de negócio, a fim de fornecer um plano prático } \\
\text { para a implementação da nova arquitetura. A metodologia é constituída de cinco etapas: mapeamento das } \\
\text { estratégias de negócio preferenciais para a adoção de web services, decomposição e documentação dos } \\
\text { processos de negócio atuais da instituição, determinação de onde e quando web services serão utilizados, } \\
\text { integração de web services, definição, decomposição e documentação de estados futuros dos web services. }\end{array}$ \\
\hline T6 & Patricia et al. [Patricia et al. 2011] & $\begin{array}{l}\text { Apresentam um meta-modelo, no qual especifica formalmente as interações necessárias entre as ativida- } \\
\text { des de análise de processo de negócio e as atividades de desenvolvimento de software para automatização } \\
\text { desses processos, a fim de extinguir a lacuna que existe entre elas. Esse modelo apresenta nove estágios: } \\
\text { Organização e Planejamento Estratégico, Levantamento e Especificação de Requisitos Orientados a Pro- } \\
\text { cessos, Levantamento dos Processos de Negócio, Modelagem dos Processos de Negócio, Modelagem } \\
\text { dos Serviços, Definição dos Componentes de Software, Implantação dos Componentes, Gerenciamento } \\
\text { e Monitoração. }\end{array}$ \\
\hline T7 & $\begin{array}{l}\text { Mondragón et } \\
\text { [Mondragón et al. 2013] }\end{array}$ & $\begin{array}{l}\text { Apresentam de forma bem estruturada uma metodologia para desenvolvimento de sistemas com apoio } \\
\text { de BPMS. Nela são apresentadas quatro fases: Previsão, Análise e Design, Construção, Execução e } \\
\text { Monitoração. Essa abordagem mescla conceitos de gerenciamento de processos de negócio com conceitos } \\
\text { de desenvolvimento de sistemas para apoiar automatização para processos. }\end{array}$ \\
\hline T8 & Prades et al. [Prades et al. 2013] & $\begin{array}{l}\text { Definem uma metodologia para realizar design e implementação de modelos de processos de negócio que } \\
\text { se utilização da notação BPMN de acordo com o padrão ANSI/ISA-95 em fábricas. O objetivo é propor } \\
\text { uma abordagem para integrar processos empresariais e de fábrica. Para isso a notação BPMN foi utilizada } \\
\text { para representar a integração desses processos e utilizada de acordo com o padrão ANSI/ISA-9. }\end{array}$ \\
\hline T10 & Herden et al. [Herden et al. 2014] & $\begin{array}{l}\text { Definem uma abordagem em BPMN para desenvolvimento de software dirigido por processo, que compre- } \\
\text { ende cinco fases: Definição do Escopo (definição dos casos de uso), Prototipação de Sistema (detalhamento } \\
\text { dos casos de uso, definição de dados, definição de telas e geração dos protótipos), Sprint de Produção } \\
\text { (integração com os dados e sistemas existentes, além de automatização de atividades), Implementação, } \\
\text { Monitoração e Implementação. }\end{array}$ \\
\hline
\end{tabular}

Tabela 1. Descrição dos Trabalhos Relacionados

o desenvolvimento de sistemas orientados a processos de negócio. A diferença abordada nesse trabalho, é que as iterações em cada fase produzem um nível de automatização de processo, no qual o cliente já pode interagir. Além disso, as iterações são em quantidade e em duração menores, da mesma forma como funciona para sprints no SCRUM. Para a primeira fase, chamada "Iniciação", o modelo analítico TO-BE, também chamado de BPD (Business Process Design) pode ser executado, porém sem riqueza de detalhes com relação as informações que trafegam no processo, apenas instanciando as atividades modeladas, para efeito de análise (simulação). Na Elaboração, telas de interação com o cliente juntamente com os dados são modelados, permitindo uma execução do processo mais rica em detalhes.

Os marcos (fases) visam a entrega de um ou vários incrementos. Um incremento pode ser o refinamento do modelo analítico TO-BE, modelo executável de um marco do processo de negócio ou do processo completo, relatório de simulação da modelagem, documento de arquitetura e etc. Uma instância do processo de desenvolvimento pode passar 


\begin{tabular}{|l|l|l|l|l|l|l|l|l|l|l|l|}
\hline & T0 & T1 & T2 & T3 & T4 & T5 & T6 & T7 & T8 & T9 & T10 \\
\hline Análise Corporativa do Processo & $\mathrm{X}$ & - & $\mathrm{X}$ & $\mathrm{X}$ & $\mathrm{X}$ & $\mathrm{X}$ & $\mathrm{X}$ & $\mathrm{X}$ & - & $\mathrm{X}$ & - \\
\hline Análise Orientada a Serviços & $\mathrm{X}$ & - & $\mathrm{X}$ & $\mathrm{X}$ & $\mathrm{X}$ & $\mathrm{X}$ & $\mathrm{X}$ & $\mathrm{X}$ & $\mathrm{X}$ & $\mathrm{X}$ & - \\
\hline Conceitos da disciplina de BPM & $\mathrm{X}$ & - & $\mathrm{X}$ & - & - & - & $\mathrm{X}$ & $\mathrm{X}$ & $\mathrm{X}$ & $\mathrm{X}$ & $\mathrm{X}$ \\
\hline Desenvolvimento com Metodologias Ágeis & $\mathrm{X}$ & - & - & - & - & - & - & - & - & - & $\mathrm{X}$ \\
\hline $\begin{array}{l}\text { Desenvolvimento de Sistema para Automatização de } \\
\text { Processos }\end{array}$ & $\mathrm{X}$ & $\mathrm{X}$ & $\mathrm{X}$ & $\mathrm{X}$ & $\mathrm{X}$ & $\mathrm{X}$ & $\mathrm{X}$ & $\mathrm{X}$ & $\mathrm{X}$ & $\mathrm{X}$ & $\mathrm{X}$ \\
\hline Desenvolvimento de Sistema com apoio de BPMS & $\mathrm{X}$ & - & $\mathrm{X}$ & - & - & - & $\mathrm{X}$ & $\mathrm{X}$ & $\mathrm{X}$ & $\mathrm{X}$ & $\mathrm{X}$ \\
\hline Design do Processo & $\mathrm{X}$ & $\mathrm{X}$ & $\mathrm{X}$ & - & $\mathrm{X}$ & $\mathrm{X}$ & $\mathrm{X}$ & $\mathrm{X}$ & $\mathrm{X}$ & $\mathrm{X}$ & $\mathrm{X}$ \\
\hline $\begin{array}{l}\text { Geração de Processo Automatizado em Múltiplas Lin- } \\
\text { guagens de Programação }\end{array}$ & - & $\mathrm{X}$ & - & - & - & - & - & - & - & - & - \\
\hline Guia de Adaptação para o Processo & - & - & - & - & - & - & - & - & - & - & - \\
\hline $\begin{array}{l}\text { Téćcicas de Estimativas Formalizadas para o Esforço } \\
\text { do Desenvolvimento }\end{array}$ & - & - & - & $\mathrm{X}$ & - & - & - & - & - & - & - \\
\hline Especificação e Design de Serviços & $\mathrm{X}$ & $\mathrm{X}$ & $\mathrm{X}$ & $\mathrm{X}$ & - & $\mathrm{X}$ & $\mathrm{X}$ & $\mathrm{X}$ & $\mathrm{X}$ & $\mathrm{X}$ & - \\
\hline $\begin{array}{l}\text { Gerência de Projeto para Desenvolvimento de Sistema } \\
\text { para Automatização de Processos }\end{array}$ & $\mathrm{X}$ & - & - & - & - & $\mathrm{X}$ & - & - & - & - & - \\
\hline Implantação de Processos Automatizados & $\mathrm{X}$ & $\mathrm{X}$ & $\mathrm{X}$ & - & - & - & $\mathrm{X}$ & $\mathrm{X}$ & $\mathrm{X}$ & $\mathrm{X}$ & $\mathrm{X}$ \\
\hline $\begin{array}{l}\text { Representação da Abordagem de Processo de Desen- } \\
\text { volvimento de Software em BPMN }\end{array}$ & $\mathrm{X}$ & - & - & - & - & - & - & - & - & - & $\mathrm{X}$ \\
\hline Testes Funcionais da Automatização do Processo & $\mathrm{X}$ & - & - & - & - & $\mathrm{X}$ & - & - & - & $\mathrm{X}$ & $\mathrm{X}$ \\
\hline
\end{tabular}

Tabela 2. Comparação entre os trabalhos relacionados e a abordagem proposta

mais de uma vez pelo mesmo marco, porém sempre desenvolvendo novas funcionalidades ou funcionalidades faltantes. A Figura 1 apresenta as atividades que são realizadas em cada marco. Um marco representa uma ou mais sprints que possui itens de backlog a serem desenvolvidos.
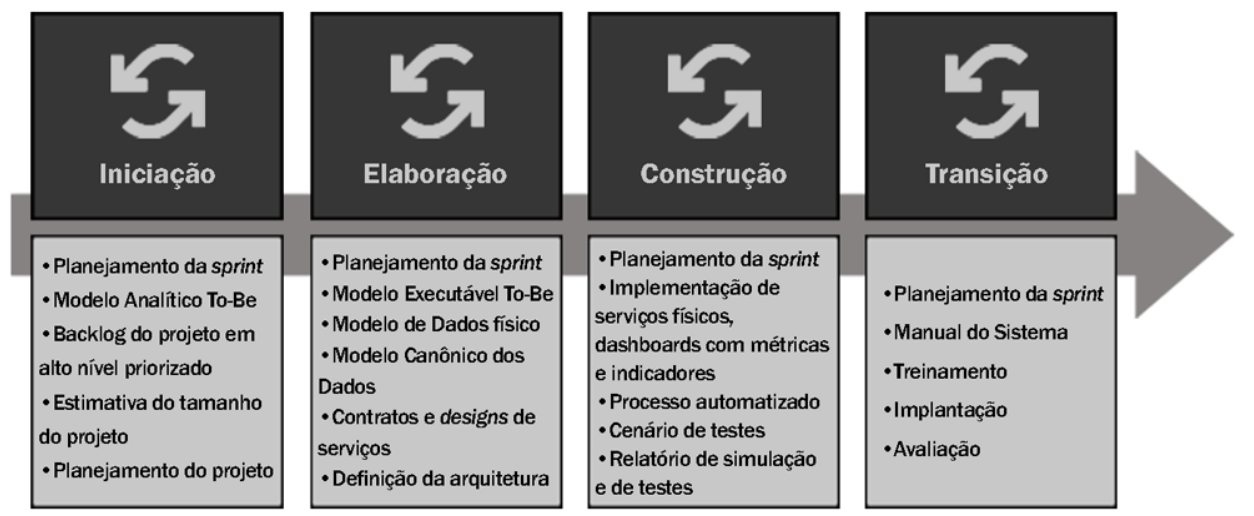

Figura 1. Sprints

\section{Abordagem Proposta}

A abordagem proposta trata o processo de desenvolvimento de aplicações de software como um processo de negócio da empresa, porém como um processo de suporte, ou seja, processo que não interage diretamente com os clientes finais. Tal processo de suporte tem o objetivo de melhorar a capacidade efetiva de realizar os processos de negócio primários na instituição.

A abordagem está representada através da notação para modelagem de processos de negócio (BPMN) [OMG 2013] e feita no BPMS chamado Bizagi Modeler [Bizagi 2013]. A representação do processo subdivide-se em subprocessos que por sua vez devem ser realizados por uma ou mais atividades (fluxos de trabalho) dentro de 
funções de negócio (áreas funcionais). As atividades são decompostas em tarefas, que devem estar representadas no fluxo também.

A definição das atividades da abordagem de processo teve como base as metodologias, ferramentas e conceitos consolidados no mercado, no que diz respeito ao desenvolvimento de sistemas tradicionais, além de considerar as áreas de conhecimento descritas no "Guia para o Gerenciamento de Processos de Negócio Corpo Comum de Conhecimento" (BPM CBOK) [ABPMP 2013]. A construção e aperfeiçoamento da abordagem foram desenvolvidos ao longo de três anos, através de projetos de pesquisa-ação que foram realizados em paralelo aos projetos de automatização de processos. A autora desse trabalho, realizou os papéis de pesquisadora e de analista de negócio, nos projetos de pesquisa-ação e automatização de processos respectivamente. A pesquisa-ação deve ser realizada preferencialmente por um papel que atua no objeto que se deseja pesquisar [Thiollent 2011].

\subsection{Pré-Requisitos}

A abordagem possui atividades e ações pré-requisitos, que precisam estar muito bem representadas e claras para todos os envolvidos no projeto. De forma prioritária, as atividades para definir as mudanças ou novos processos e as necessidades de automatização devem ser geradas por parte da Diretoria da Organização ou por Unidades de Negócio, desde que estejam alinhados com o planejamento estratégico. A tradução dessas necessidades definidas na estratégia em ações é o desafio de grande maioria dos projetos. Essas atividades estão ilustradas na Figura 2.

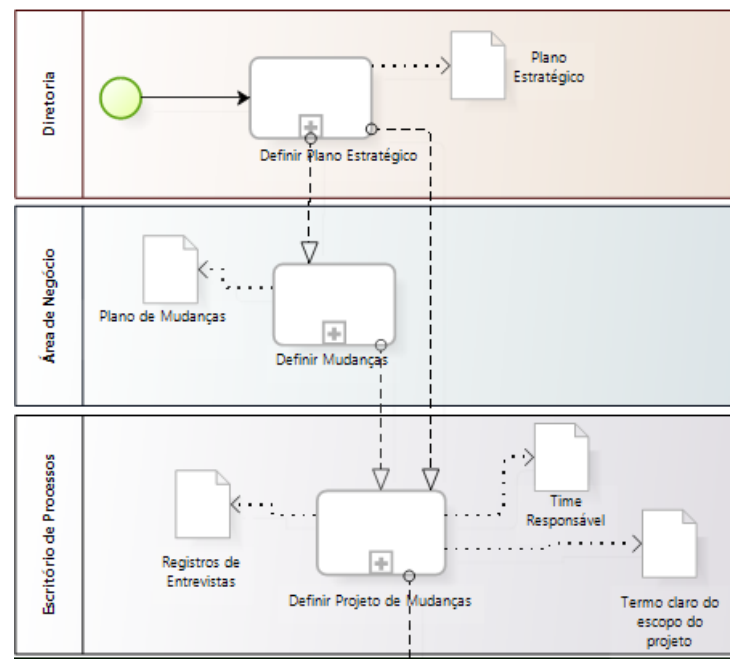

Figura 2. Atividades necessárias ao Desenvolvimento de Solução de
Automatização para Processos

Após essas atividades, uma análise corporativa deve ser realizada a fim de que os problemas atuais do processo (pontos fracos, restrições e defeitos), a amplitude de transformação do processo, necessidade do negócio de forma priorizada, o escopo da solução, as capacidades requeridas, as restrições, o caso de negócio, um vocabulário de negócio comum e a visão do projeto sejam definidos para que, só então, uma solução para atender o plano estratégico ou plano de mudanças seja definida. 
As próximas seções descrevem os marcos definidos para a abordagem do processo de desenvolvimento de automatização para processos de negócio.

\subsection{Iniciação}

Essa fase possui o objetivo de realizar análise e especificação de requisitos, obtendo uma visão mais sistemática da orquestração das atividades através da ferramenta de BPMS. Nela os requisitos de automatização do processo são organizados, priorizados, especificados, modelados e verificados.

A modelagem, neste nível, deve retratar os padrões para ramificação e unificação de processos, bem como padrões para tratamento de exceções. Inclui a ordenação das atividades em um fluxo com base nos relacionamentos das atividades, identificação e associação de competências e processos de suporte necessários para os processos. Além disso, deve considerar o nível de fluxo de processo (visão interfuncional) e o nível de fluxo de trabalho (visão intrafuncional). Funciona como uma sprint zero [ABPMP 2013]. Principais pontos que precisam ser definidos na sprint zero são:

- Quem serão os envolvidos no projeto?

- Como o projeto será organizado? (Ex.: plano de comunicação e qual processo será utilizado)

- Quais são os principais riscos e impedimentos?

- Qual o escopo do projeto?

- O que será desenvolvido? (obter requisitos de negócio e elaborar backlog do produto priorizado, backlog selecionado e backlog da sprint.

- Quais pessoas o projeto precisa para desenvolver determinada funcionalidade?

- Para quando precisa-se entregar algo?

Após a avaliação positiva de continuidade do atendimento da demanda, atividades de análise e especificação de requisitos, priorização e planejamento de entregas funcionais e operacionais programadas de versões do processo já podem ser definidas. A implantação de um sistema para apoiar processos de negócio extensos e complexos precisa ser feita de forma gradativa. Pois, ao iniciar contemplando todas as funcionalidades do projeto de uma só vez, corre-se um grande risco de, em vez do sistema auxiliar a versão do processo que será implantada poderá causar grandes transtornos.

A Iniciação, em geral, possui muitas iterações quando o projeto possui algumas das características a seguir:

- Projeto possui estimativa muito grande (acima de seis meses de duração);

- Escopo e limites difícil de definir;

- Muitos envolvidos com necessidades conflitantes e relações complexas;

- Principais riscos técnicos exigem a construção de protótipo ou prova de conceito;

- Dificuldade das áreas envolvidas no processo liberarem pessoas para contribuir com a especificação do novo modelo de processo;

A principal atividade da fase de Iniciação é "Analisar Requisitos" (Tabela 3). Consiste principalmente, para que os recursos de TI (analistas de sistemas, arquitetos de sistema/serviços, gerente de projeto e etc) que foram alocados para o projeto e, portanto, adicionados ao time responsável pelo projeto possam aprofundar-se nas necessidades e resoluções de problemas para o novo processo. A técnica escolhida para o aprofundamento dessas informações foi o workshop estruturado. 


\begin{tabular}{|c|c|}
\hline \multicolumn{2}{|r|}{ Atividade: Analisar Requisitos } \\
\hline Descrição: & $\begin{array}{l}\text { Estabelecer entendimento comum a respeito da demanda entre todos do time responsável pelo projeto, além de realizar o refina- } \\
\text { mento da modelagem analítica da solução para o processo de negócio. }\end{array}$ \\
\hline \multirow[t]{6}{*}{ Tarefas: } & $\begin{array}{l}\text { Para definir se a modelagem possui os elementos necessários e bem definidos para um bom entendimento comum, como analista } \\
\text { de negócio, devo aplicar checklist na modelagem AS-IS e modelo analítico TO-BE. }\end{array}$ \\
\hline & $\begin{array}{l}\text { Para realizar workshops dirigidos e estruturados cujo objetivo é apresentar o resultado da análise, refinar modelagem analítica } \\
\text { e todos os demais artefatos necessários para subsidiar a definição da transformação do processo, como gerente do projeto, devo } \\
\text { convocar pessoas responsáveis pelo processo. }\end{array}$ \\
\hline & $\begin{array}{l}\text { Para realizar o repasse das informações de análise, como analista do processo com apoio de especialistas de negócio, devo } \\
\text { apresentar a modelagem AS-IS, modelo analítico TO-BE e todas as informações obtidas na análise. }\end{array}$ \\
\hline & $\begin{array}{l}\text { Para retirar as dúvidas e prospectar soluções tecnológicas para os problemas apresentados, como analista de negócio com apoio } \\
\text { de analista de sistema e arquiteto de serviços, devo aplicar questionário de dúvidas e, de posse das informações, se necessário, } \\
\text { devo refinar a modelagem analítica. }\end{array}$ \\
\hline & $\begin{array}{l}\text { Para obter a estimativa do tamanho total da transformação do projeto determinada pelos analistas de sistemas/desenvolvedor, } \\
\text { como gerente de projeto, devo gerar o backlog inicial do produto, contendo a visão inicial do desenvolvimento da solução. }\end{array}$ \\
\hline & $\begin{array}{l}\text { Para decidir entre estabelecer uma baseline com os artefatos até então já produzidos e cancelar a demanda, como gerente do } \\
\text { projeto, devo negociar e obter acordo entre todos os interessados na solução em relação ao escopo e estimativa do projeto. }\end{array}$ \\
\hline Responsáveis: & $\begin{array}{l}\text { Time Responsável pelo Projeto Estratégico. O time deve ser interfuncional: especialistas do negócio (liderança executiva, } \\
\text { gerência intermediária e executores do processo), analista de processo, gerente de processo, gerente do projeto, dono do pro- } \\
\text { cesso, analista de negócio, analista de sistema e arquiteto de serviços. }\end{array}$ \\
\hline Artefato de Entrada: & Modelo AS-IS, modelo analítico TO-BE e documentos contendo todas as informações obtidas com a realização da análise. \\
\hline Artefato de Saída: & $\begin{array}{l}\text { Checklists de conformidade aplicado aos modelos entregues, modelo analítico TO-BE refinado, especificação de métricas e } \\
\text { indicadores, regras de negócio, backlog da automatização do processo priorizado, estimativa do tamanho do projeto e termo de } \\
\text { aceite. }\end{array}$ \\
\hline
\end{tabular}

Tabela 3. Analisar Requisitos

\subsection{Elaboração}

Um dos principais propósitos da fase de Elaboração é evoluir o modelo analítico TO-BE do processo para um primeiro nível do modelo executável, considerando apenas o que foi priorizado e selecionado para compor o backlog da sprint. Para isso, é preciso fazer um estudo dos serviços disponíveis na instituição que poderão ser reutilizados ou que terão de ser construído pensando nessa estrutura de organização de serviços.

O modelo é evoluído pelo analista de negócio que pode consultar especialistas do negócio, analista de processo e arquiteto de serviços. Um primeiro nível do modelo executável é um estágio intermediário para se atingir a modelagem completa de execução do processo. Os detalhes a mais a serem representados são informações acerca do funcionamento das atividades do processo, que visam resolver as questões descobertas na análise e consequentemente atender aos objetivos da estratégia traçada. Esse modelo se aproxima do desenho final de processos, pois já possui uma definição formal de objetivos e entregáveis, organização das atividades e regras necessárias para produzir um resultado desejado. As principais atividades desse marco são: "Desenhar a solução" (Tabela 4) e "Realizar Análise Orientada a Serviço", conforme ilustrado na Figura 3.

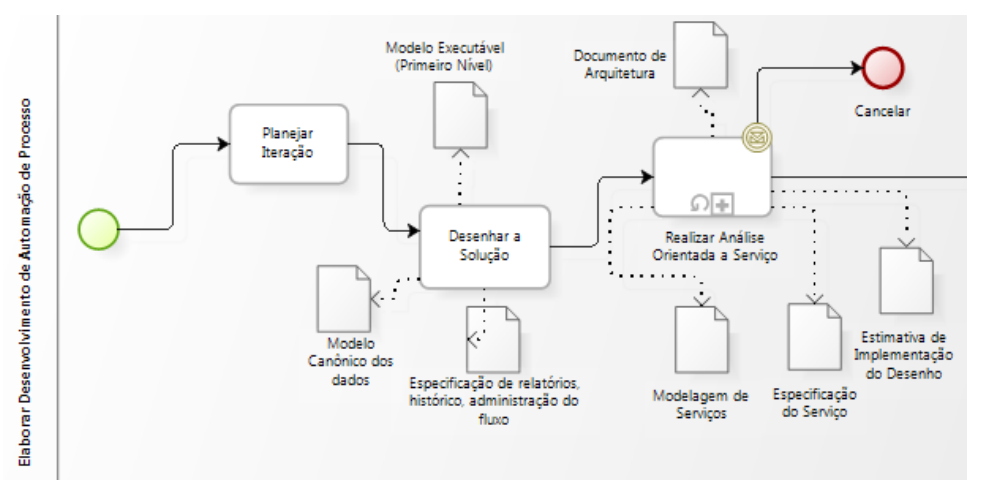

Figura 3. Atividades de Elaboração 
Durante a elaboração do primeiro nível do modelo executável alguns itens-chave com relação ao processo e funcionalidades sistêmicas são considerados:

- Definir e modelar os objetos/dados de negócio que serão trafegados ao longo da execução do processo, de preferência estabelecer um modelo canônico para esses dados de negócio considerando todos os outros dados dentro da instituição. Para isso, é preciso identificar a origem dos dados, ou qual área é dona da informação.

- Apontar os dados de entrada e de saída de cada atividade, além de definir variáveis internas de cada atividade.

- Criar serviços de atividades humanas e configurá-los nas atividades. Aqui é interessante analisar se as interfaces humanas serão reutilizáveis.

- Criar as interfaces (telas) de entrada de dados das atividades, de forma componentizada.

Alguns elementos da notação BPMN são sugeridos para serem analisados e desenhados com relação a implementação neste momento, são eles: eventos de envio, eventos de recebimento, tarefa de regra de negócio, tarefa de usuário global, subprocesso ad-hoc, subprocesso transacional, atividade de chamada/referência, atividade de loop, atividade de múltipla instância, gateway inclusivo, fluxo de sequência condicional, gateway de evento, início condicional, início por sinal, fim por erro, fim por sinal, timers (temporizadores), eventos de interrupção e não interruptivos em limites (Interrupting and NonInterrupting Boundary) [CAPOTE 2011].

Além disso, demais funcionalidades de relatório, administração do fluxo de negócio, métricas, indicadores e informações para compor histórico também devem ser levantadas nesse momento, principalmente para identificar e mapear os dados a serem utilizados nessas funcionalidades e qual é a origem deles.

\begin{tabular}{|l|l|}
\hline \multicolumn{2}{|l|}{ Atividade: Desenhar a Solução } \\
\hline Descrição: & $\begin{array}{l}\text { Elaborar o primeiro nível do modelo executável. Consiste em definir e modelar os objetos (dados) de negócio que serão trafegados } \\
\text { ao longo da execução do processo, criar serviços de atividades humanas e configurá-los nas atividades, criar as interfaces (telas) } \\
\text { de entrada de dados das atividades, assim como identificar a origem dos dados. }\end{array}$ \\
\hline Tarefas: & $\begin{array}{l}\text { Para especificar e modelar os objetos de negócio (modelo canônico), criar as interfaces (telas) de entrada de dados das atividades, } \\
\text { configurar dados de entrada e saída das atividades, criar serviços de atividades humanas e configurá-los nas atividades, assim } \\
\text { como configurar demais elementos no processo, como analista de negócio, quando preciso, devo convocar especialistas de } \\
\text { negócio e analista de processo. Além disso, para apoiar a administração do fluxo de negócio deve ser feito um levantamento de } \\
\text { dados, além de métricas e indicadores que irão compor relatórios e histórico. }\end{array}$ \\
\hline Responsáveis: & $\begin{array}{l}\text { Time Responsável pelo Projeto Estratégico. O time deve ser interfuncional: especialistas do negócio (liderança executiva, } \\
\text { gerência intermediária e executores do processo), analista de processo, gerente de processo, analista de negócio. }\end{array}$ \\
\hline $\begin{array}{l}\text { Artefato de En- } \\
\text { trada: }\end{array}$ & $\begin{array}{l}\text { Modelo analítico TO-BE e documentos contendo todas as informações obtidas com a realização da análise (necessidades de } \\
\text { negócio capacidades requeridas, escopo da solução, amplitude da transformação do processo e etc). }\end{array}$ \\
\hline Artefato de Saída: & Modelo Executável (primeiro nível), modelo canônico dos dados e demais especificações técnicas. \\
\hline
\end{tabular}

\section{Tabela 4. Desenhar a Solução}

O modelo analítico provê um bom nível de informação necessária para que se possa compreender toda a semântica do domínio da automatização. Alcançar a semântica da automatização do processo de forma consistente representa um dos grandes desafios para a criação de SOA. Dessa forma, o arquiteto de sistema/serviços juntamente com o analista de negócio precisam identificar toda a semântica de automatização e metadados que existem no domínio e, portanto, definir o que permite lidar adequadamente com esses dados. A compreensão da semântica da automatização estabelece o caminho e a forma em que as interfaces de serviço particulares são peculiares, diferenciando-se apenas pelas propriedades que exercem no processo de negócio [Thomas 2005]. Esses passos são realizados na atividade "Realizar Análise Orientada a Serviço". 


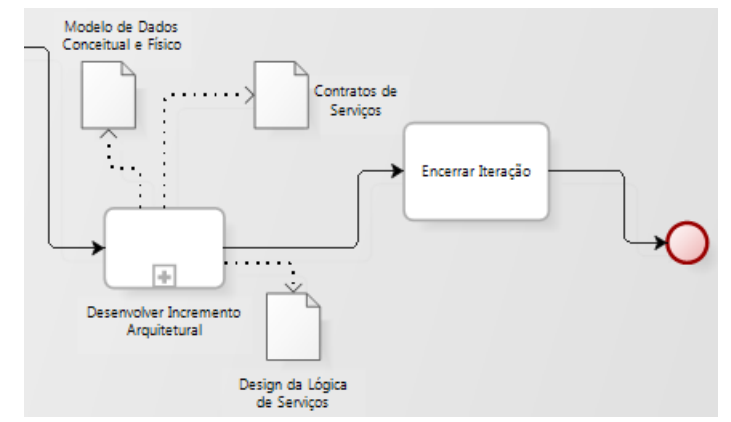

Figura 4. Atividades finais da Elaboração

Após a análise orientada a serviço, o primeiro incremento da solução com foco na arquitetura é elaborado, conforme ilustrado na Figura 4. A primeira tarefa é realizar o design orientado a serviços da solução. O design orientado a serviços utiliza o conjunto de serviços candidatos predefinidos como ponto inicial e pelo qual serão modelados em contratos de serviços físicos reais. A diferença entre um candidato a serviço (conceitual) e o serviço físico é que o primeiro representa um serviço conceitual, ou seja, é do ponto de vista de negócio, o último, refere-se a um serviço do ponto de vista técnico, como será implementado, ou como será do ponto de vista físico.

\subsection{Construção}

A fase de Construção trata do desenvolvimento do projeto com base na arquitetura definida na fase de Elaboração. Apenas nessa fase, após análise técnica das funcionalidades, tem-se a visão mais clara de como as funcionalidades devem ser implementadas. Porém, alguns requisitos ainda podem ser refinados para a realização de um melhor desenvolvimento da solução, conforme ilustrado na Figura 5.

O propósito desta fase, no ciclo de vida do projeto, é ter um incremento do produto pronto para ser implantado durante a fase de transição. O planejamento de entregas do projeto define se o incremento pode ser uma versão completa ou parte da solução. As funcionalidades que serão desenvolvidas e testadas aqui devem estar de acordo com a especificação e arquitetura definidas anteriormente.

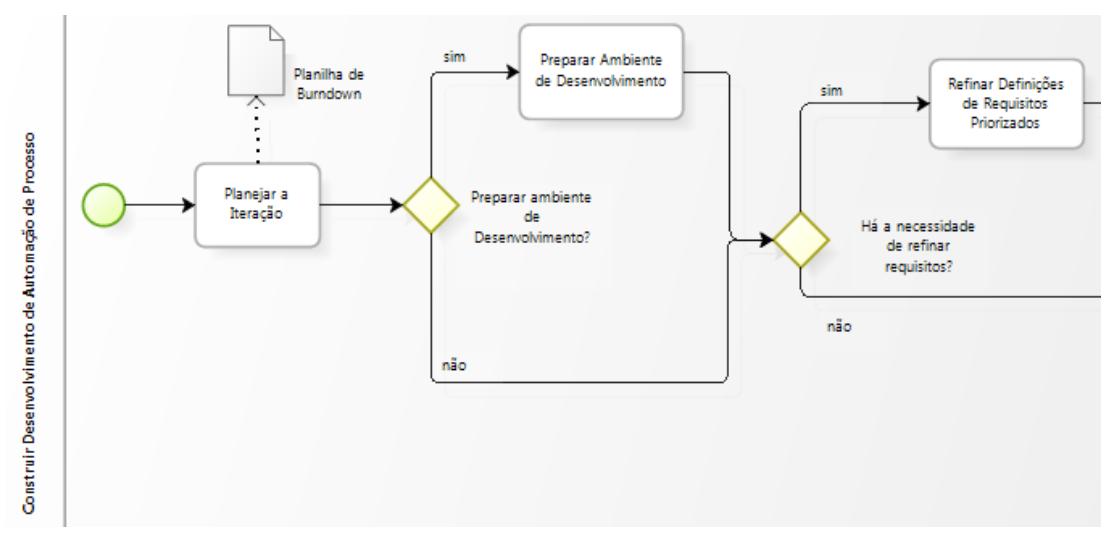

Figura 5. Atividades de Construção 
Os objetivos principais na fase de Construção são:

- Desenvolver a solução: iterativamente desenvolver o incremento do produto/projeto especificado nas fases anteriores e deixá-lo pronto para a transição para o usuário final.

- Minimizar os custos: foco na otimização de recursos, evitando retrabalho e atividades desnecessárias.

- Atingir certo grau de paralelismo entre os times de desenvolvimento: planejar o uso dos recursos em sinergia com o foco na minimização dos custos e com a priorização dos itens a serem desenvolvidos a cada iteração.

- Atingir a versão para entrega com qualidade adequada, rapidez e eficiência: agregar valor para os clientes.

Os próximos passos a serem realizados na fase de Construção estão ilustrados na Figura 6. A primeira atividade ilustrada na figura a se fazer é desenvolver incrementos da solução que tornarão o processo totalmente automatizado.

Os incrementos a serem desenvolvidos nessa fase são:

- Refinamento do design e lógica de comportamento nas telas.

- Codificação de serviços e operações de integrações, eventos, rotinas e regras de negócio.

- Codificação de funcionalidades administrativas do fluxo.

- Cenários de teste.

- Relatório de Simulações e de testes.

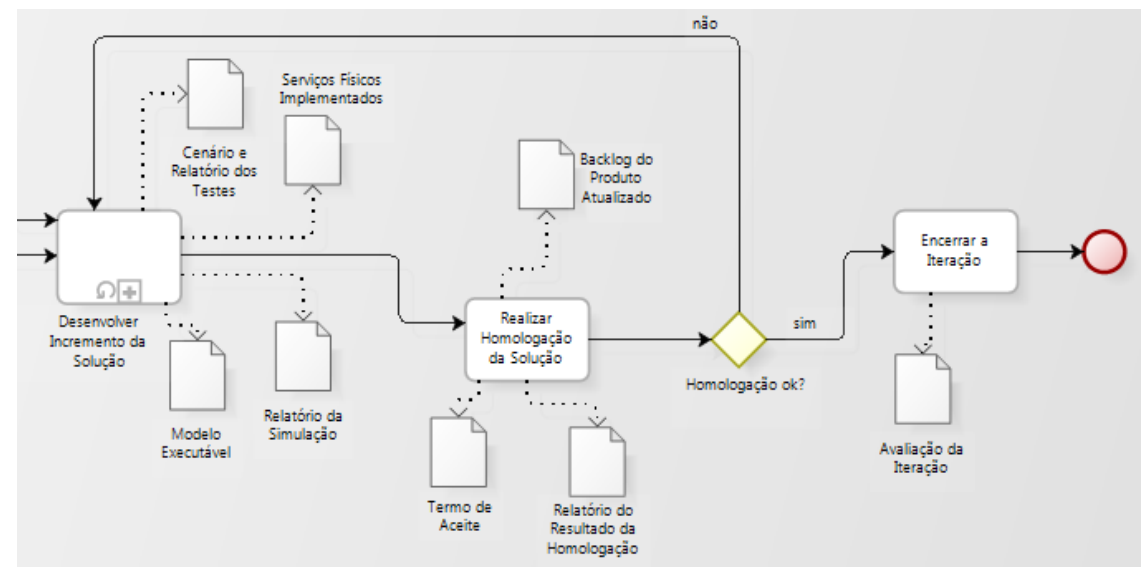

Figura 6. Atividades finais da Construção

Nesse ponto, o modelo executável do processo de negócio é ainda mais refinado até ficar totalmente automatizado.

\subsection{Transição}

Para o marco de Transição, todos os problemas estruturais mais graves devem ter sido trabalhados nas fases anteriores do ciclo de vida do projeto. Os principais objetivos do projeto devem ter sido atendidos e o mesmo deve estar em uma posição de fechamento. Em alguns casos, o fim do ciclo de vida atual pode coincidir com o início de outro ciclo 
de vida no mesmo produto, conduzindo à nova geração ou versão do produto. Isto ocorre principalmente em caso de produtos já existentes que sofrem evolução. Para outros projetos, o fim da Transição pode coincidir com uma liberação total dos artefatos para os responsáveis pela operação, manutenção e melhorias no sistema, concluindo o projeto.

Dentre os principais objetivos da fase de Transição podemos citar:

1. Elaboração de manual de instrução.

2. Treinamento de usuários e time de manutenção. Transmissão de informações para os times de operação e manutenção.

3. Obtenção do consentimento dos envolvidos em relação à release entregue e da aderência da versão com os termos da especificação de requisitos. É gerada a baseline final de todos os artefatos do projeto.

4. Documentar as lições aprendidas no projeto.

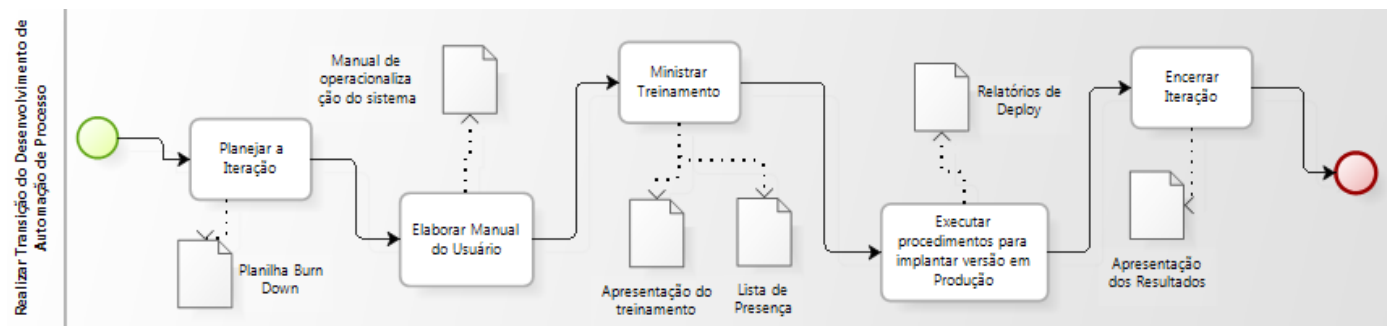

Figura 7. Atividades da Transição

A Figura 7 descreve o fluxo completo das atividades da fase de Transição.

Assim como nas fases anteriores (Elaboração e Construção), ao longo de toda a fase de Transição, pode-se realizar uma atividade para avaliar a qualidade. Esta atividade é executada para avaliar os artefatos desenvolvidos, em uma amostra de instâncias de processos a fim de descobrir se foram gerados em conformidade com os padrões de qualidade definidos, com foco na implantação do novo processo.

As quatro fases (Iniciação, Elaboração Construção e Transição) descritas compõem o processo de suporte de desenvolvimento de sistema que apoia a execução de um processo de negócio. Vale ressaltar que as funcionalidades tecnológicas desenvolvidas com apoio de um BPMS por si só não irão fazer com que o processo de negócio seja realizado de forma plena.

A preocupação efetiva com as pessoas que trabalham na execução do processo é de grande importância. Muitas melhorias falham em serem implementadas, por acharem que apenas a tecnologia por si mudará o processo. Na verdade, as pessoas que mudam o modo de agir e assim mudam o processo. Os fatores humanos, culturais e comportamentais influenciam fortemente uma implementação de processo e de sistema que apoiam tais processos. Em especial, ressalta-se a ideia de identificar os profissionais certos nas unidades envolvidas e motivá-los para criação e transição para o novo processo.

É preciso reconhecer que pelo menos 50\% das oportunidades de melhorias em processos passam por tecnologia, sendo fundamental integrar conhecimentos de TI e negócio para traduzir como tais melhorias podem ser operacionalizadas [ABPMP 2013]. 


\section{Validação da Abordagem e Conclusão}

O método de Pesquisa Ação utilizado para desenvolver e validar a abordagem, provê análise do processo de desenvolvimento de software de forma orientada a processo, assim como constrói teorias dentro de um contexto democrático e organizacional. Dessa forma, dois ciclos de Pesquisa Ação foram realizados, contando com a participação das pessoas pertencentes à célula de suporte a processos de TI, pessoas de negócio especialistas no assunto, dono do processo, analista de processos, de negócios e de sistemas. Com isso, a Pesquisa Ação teve uma abordagem participativa, menos formalizada, porém mais efetiva e personalizada, pois envolveu participação de pessoas executoras do projeto e pesquisadores que também desempenharam atividades dentro do projeto, ou seja, que estiveram envolvidos e comprometidos.

A metodologia utilizada para os projetos de pesquisa-ação considerou a existência de no mínimo, quatro grandes fases [Thiollent 2011]:

1. Fase Exploratória: É feito uma investigação a respeito de uma determinada situação por parte dos pesquisadores e de membros da organização para detectar problemas, atores envolvidos, capacidades de ação e tipos de ação possível.

2. Pesquisa Aprofundada: É feito uma pesquisa aprofundada a respeito da situação investigada em diversos tipos de instrumentos de coleta de dados. Os dados são discutidos e progressivamente interpretados.

3. Fase de Ação: Consiste em difundir os resultados, definir objetivos alcançáveis por meio de ações concretas e apresentar propostas que poderão ser negociadas com base nas investigações em curso.

4. Fase de Avaliação: Consiste em observar, redirecionar o que realmente acontece e resgatar o conhecimento produzido no decorrer do processo. Realiza-se uma validação com as pessoas envolvidas no projeto.

Os problemas detectados e investigados nas fases "Exploratória" e "Aprofundada" dos projetos de Pesquisa-Ação realizados foram reunidos na Tabela 5.

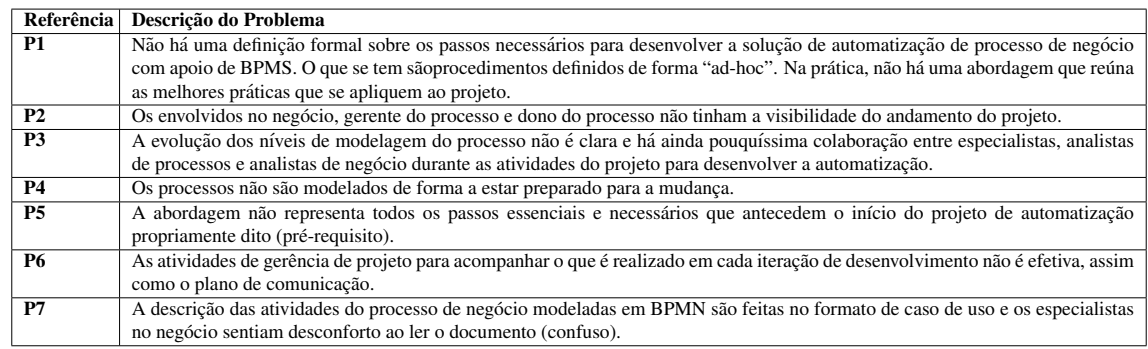

Tabela 5. Problemas

Na Fase de Ação, para cada problema apresentado, uma ação foi tomada com objetivo de solucioná-lo. A Tabela 6 reúne as ações tomadas para cada problema relacionado.

As diferentes práticas (Técnicas de desenvolvimento ágil, RUP, SOA, BPM, SCRUM) utilizadas e a representação do processo de desenvolvimento de software em BPMN proporcionaram alinhamento de conceitos que, anteriormente, os envolvidos no 


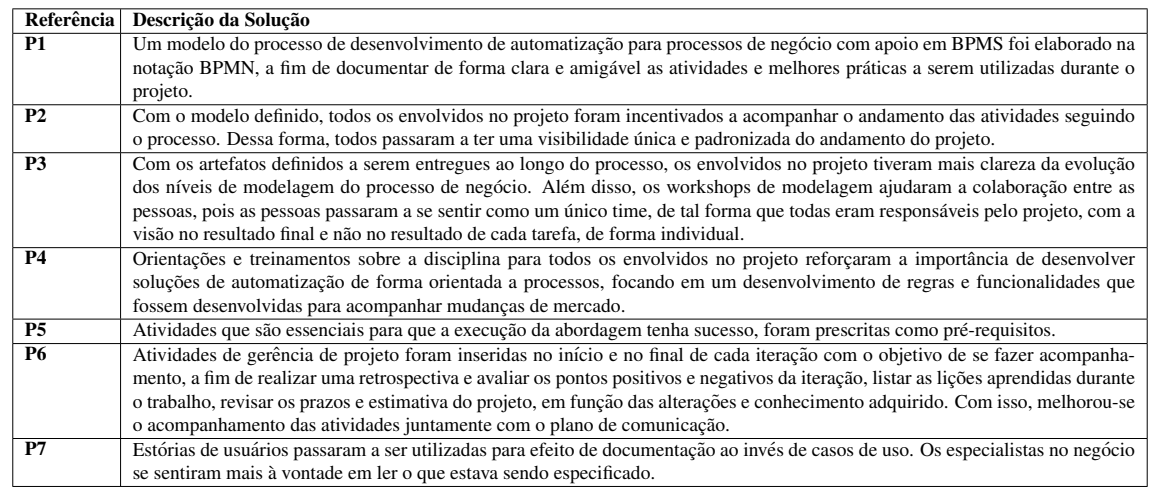

Tabela 6. Soluções

projeto não sabiam como combinar e quais deles melhor se aplicavam. Pode-se perceber que foram obtidos mais acertos na automatização do processo e menos problemas de comunicação entre os profissionais. Ou seja, obteve-se dentre os benefícios o entendimento comum dos papéis de cada envolvido no projeto, tarefas e práticas para serem, futuramente, utilizadas ao longo dos demais projetos.

A abordagem representada na notação BPMN que, normalmente, é utilizada para representar processos de negócio diferentemente de processos de TI, foi um grande diferencial para determinar os marcos/fases do processo, para colher métricas do processo de desenvolvimento de software orientado a processo e foi o primeiro passo para possibilitar análise para melhorias contínuas, proporcionando um produto com mais qualidade.

A realização de projetos de pesquisa-ação foi fundamental na construção de um processo aderente à realidade da empresa, de forma alinhada com os objetivos organizacionais.

\section{Referências}

ABPMP (2013). Guia para o Gerenciamento de Processos de Negócio Corpo Comum de Conhecimento - BPM CBOK, volume versão 3.0 - $1^{\text {a }}$ Edição.

An, L., Yan, J., and Tong, L. (2008). Methodology for web services adoption based on technology adoption theory and business process analyses. Tsinghua Science Techno$\log y, 13(3): 383-389$.

Audy, J. and Prikladnicki, R. (2007). Desenvolvimento Distribuído de Software: Desenvolvimento de software com equipes distribuídas.

Bizagi (2002-2013). http: / /www.bizagi.com/.

CAPOTE, G. (2011). Guia para formação de analistas de processos $1^{\mathrm{a}}$ edição.

Delgado, A., Weber, B., Ruiz, F., Garcia-Rodríguez De Guzmán, I., and Piattini, M. (2014). An integrated approach based on execution measures for the continuous improvement of business processes realized by services. Information and Software Technology, 56(2):134-162.

Erl, T. (2008). SOA: Principles of Service Design, volume 1. Prentice Hall Upper Saddle River. 
Harmon, P. (2010). Business Process Change: a guide for business managers and BPM and Six Sigma Professionals. Morgan Kaufmann.

Herbsleb, J. D., Mockus, A., Finholt, T. A., and Grinter, R. E. (2001). An empirical study of global software development: distance and speed. In Proceedings of the 23rd international conference on software engineering, pages 81-90. IEEE Computer Society.

Herden, A., Farias, P., de Andrade, P., and Albuquerque, A. (2014). Agile pdd-one approach to software development using bpmn. In 11th International Conference Applied Computing, Porto, Portugal.

Karastoyanova, D. (2004). A methodology for development of web service-based business processes. Proceedings of AWESOS.

Kruchten, P. (2004). The rational unified process: an introduction. Addison-Wesley Professional.

Mamaghani, N. D., Mousavi, F., Hakamizadeh, F., and Sadeghi, M. (2010). Proposed combined framework of soa and rup. In Information Sciences and Interaction Sciences (ICIS), 2010 3rd International Conference on, pages 346-351. IEEE.

Mondragón, M., Mora, M., Garza, L., Álvarez, F., Rodríguez, L., and Duran-Limon, H. A. (2013). Toward a well-structured development methodology for business processoriented software systems based on services. Procedia Technology, 9:351-360.

OMG (1997-2013). http: / / www . bpmn . org/.

Papazoglou, M. P. and Van Den Heuvel, W.-J. (2007). Business process development life cycle methodology. Communications of the ACM, 50(10):79-85.

Patricia, B., Perez, G., Giandini, R., and Diaz, J. (2011). Process-service interactions using a soa-bpm-based methodology. In Proceedings of the 2011 30th International Conference of the Chilean Computer Science Society, pages 100-107. IEEE Computer Society.

Prades, L., Romero, F., Estruch, A., Garcia-Dominguez, A., and Serrano, J. (2013). Defining a methodology to design and implement business process models in bpmn according to the standard ansi/isa-95 in a manufacturing enterprise. Procedia Engineering, 63:115-122.

Schwaber, K. and Beedle, M. (2001). Agile Software Development with Scrum. Prentice Hall PTR, Upper Saddle River, NJ, USA, 1st edition.

Thiollent, M. (2011). Metodologia da pesquisa-ação. In Metodologia da pesquisa-ação. Cortez.

Thomas, E. (2005). Service-oriented architecture: concepts, technology, and design. Prentice Hall, 31:W3C.

Vidales, M. Á. S., García, A. H., and Aguilar, L. J. (2006). Una recomendación basada en mda, bpm y soa para el desarrollo de software a partir de procesos de negocio en un contexto de negocio bajo demanda. University Pontificia of Salamanca. 